\title{
Would the STRASS study be an "unbroken" paradigm for retroperitoneal sarcomas?
}

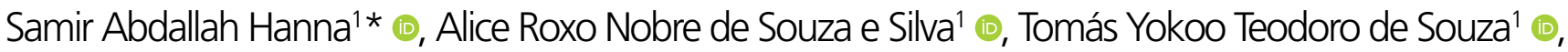 \\ Felipe Cicci Farinha Restini ${ }^{1} \odot$, David Rodrigues Ferreira Neto ${ }^{1} \odot{ }^{\circ}$, Letícia Hernandes de Brito ${ }^{1} \odot$,

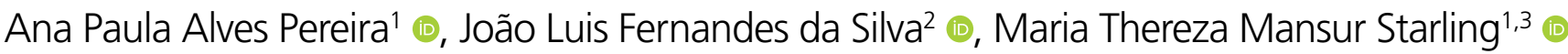

The authors of the study that was recently published in the journal Lancet Oncology ${ }^{1}$ deserve praise and compliments for the difficulty in conducting a phase III, open, multicenter, randomized study, particularly in the case of retroperitoneal sarcomas (an uncommon and heterogeneous entity). Since 30 years ago, from a small randomized series ${ }^{2}$ exploring the role of intraoperative radiotherapy in retroperitoneal sarcomas, no strong evidence for radiotherapy as a suitable treatment for this disease has been observed. In the medical field, our decisions are usually based on Level I Evidence. As radio-oncologists, however, the following important aspects could be pointed out from this study:

1. The construction of a "composite" primary end point, including local and distant failures, as well as anesthetic and surgical issues (ASA 3 intraoperatively, peritoneal sarcomatosis, incomplete resection and exclusion of patients from the study, early imaging assessment for surgical adequacy) seemed to be audacious and innovative. However, the study was designed to demonstrate a $20 \%$ "superiority" in the neoadjuvant radiotherapy arm of this purpose, which may seem too radical, taking into consideration "only" 260 cumulative patients. The achievement of statistical significance was further hampered by the low compliance with the recommended protocol, in which only $65 \%$ of patients adequately completed the therapeutic plan. In addition, there was great heterogeneity among the treatment methods, as $26 \%$ of patients deviated in their protocols.

Reductions in recurrence can generate benefits in the survival of these patients. With due respect to comparisons between prospective randomized studies and retrospective publications, Nussbaum et al. ${ }^{3}$ demonstrated the benefits in survival for neoadjuvant radiotherapy "skipping" recurrence evaluations in patients with retroperitoneal sarcomas by means of National Cancer Database analyses. This required a sample of 563 patients. Perhaps, the authors of STRASS could have kept local recurrence as an end point more directly and clearly related to the objective of the study (locoregional treatments). Moreover, even if the analysis of local recurrences was not envisaged, the local recurrence rate observed by the authors was almost half of that in irradiated patients, thus the need to separate local recurrences as a single end point.

2. The early response evaluation (by means of images at 2 weeks postradiotherapy) for resectability purposes and verification of the RECIST criteria is unclear. As there was no time for a therapeutic response to radiotherapy treatment, the evaluation of resectability should not have been performed at this moment. Moreover, cases presenting lesion enlargement based on RECIST criteria were already characterized as disease progression, not being initially referred to surgery. The response rate is best stipulated by pathological characteristics plus RECIST criteria. This aspect has been previously studied ${ }^{4,5}$ particularly in extremity sarcomas (among other neoplasms), where the clinical and pathological response rates are directly correlated with the time between neoadjuvant treatments and surgery, although radiological alterations mentioned above are not sufficient to confirm disease progression in this scenario. After radiotherapy, particularly in the earlier period, significant vasodilatation and inflammation of the irradiated tissue occurs, leading to a possible increase in its volume, thus being

\footnotetext{
'Hospital Sírio-Libanês, Departamento de Radio-Oncologia - São Paulo (SP), Brazil.

${ }^{2}$ Hospital Sírio-Libanês, Departamento de Radioterapia - São Paulo (SP), Brazil.

${ }^{3}$ Instituto do Câncer do Estado de São Paulo - São Paulo (SP), Brazil.

*Corresponding author: samir.hanna@hsl.org.br

Conflicts of interest: the authors declare there is no conflicts of interest. Funding: none.

Received on March 30, 2021. Accepted on April 26, 2021.
} 
equivocal to veto the surgical possibility-the curative pillar of the retroperitoneal sarcoma. How did the authors evaluate some patients (15/19) who presented "disease progression," but had been successfully operated on with free margins? Why were these patients excluded from the analysis?

3. Even though it was not planned within the same study (analysis in STRASS $2^{6}$ ), it would be worth knowing the effects of systemic therapies in the neoadjuvant setting, including their interactions with radiotherapy. Some drugs have interesting response rates depending on the histological subtype, and exploring this strategy could change the current treatment patterns.

4. The dose of 50 Gy over 5 weeks has been the standard "generic" radiotherapy dose in the neoadjuvant setting, including its adoption in the study by O'Sullivan et al. ${ }^{7}$, involving patients with soft-tissue sarcomas of the extremities, primarily analyzed as outcomes of scar complications-not oncological outcomes. Thus, the neoplastic dose employed by the STRASS researchers may be questionable if considered in comparison with this Canadian study. Sarcomas are radioresistant tumors. Would it be possible, when using intensity-modulated radiation therapy (used in $95 \%$ of STRASS patients), to use higher doses applied to the tumor? Would it not be worthwhile to apply dose escalation in areas with supposedly greater difficulty of resection, preferably after a multidisciplinary discussion with the surgeons?

5. In view of the difficulty in obtaining a homogeneous sample of various histological subtypes, why did the authors not reduce the range of inclusion of the histological subtype in the study? For example, they could have considered only liposarcomas and leiomyosarcomas. Perhaps, the study design could have been modified according to the less heterogeneous sample (even taking into consideration that most patients were liposarcoma cases). It is important to remember that the main benefit of radiotherapy for patients with retroperitoneal sarcoma is the improvement in the local control of the disease. Such a role becomes even more relevant in patients whose surgical outcome is not ideal, as in cases of resections with compromised margins (R1) or macroscopic residual disease (R2). Thus, the analysis of the results of the subgroups of this study according to the degree of resection is fundamental, and to understand that the selection of the best candidates for surgery may result in the exclusion of those who benefit the most from the local control resulting from radiotherapy. In an interim analysis performed for sensitivity assessment of the study, early local progression was not considered a primary end point for patients who subsequently achieved complete surgical resection. Most patients (75\%) had liposarcoma (well-differentiated $33.1 \%$, dedifferentiated 39.5\%), whereas leiomyosarcoma accounted for
$14.3 \%$ of cases. After a median 43-month follow-up, local recurrence-free survival was $60.4 \%$ versus $58.7 \%$ for the irradiated versus nonirradiated group $(\mathrm{p}=0.95)$. After the interim analysis, this end point could be considered only in the liposarcoma subgroup ${ }^{8}$.

6 . From the methodological point of view, one can question the degree of radiotherapy treatment described in the procedures of the publication. Retroperitoneal sarcomas are usually diagnosed with large volumes, and, in fact, the mean size identified in the study was $16 \mathrm{~cm}$. However, a clinical treatment volume (CTV) of 5-6 cm was stipulated, to which a planning margin (PTV) of 9-12 cm was added, depending on the axis in question. It is important to note that the study does not specify whether there was editing of CTVs according to the risk organs, as well as whether there were dose limits recommended for them. This issue becomes even more relevant when we face high rates of toxicities, especially gastrointestinal and hematological, which may indicate excessive irradiation of the abdominal cavity and bone marrow, respectively. Another important methodological flaw is the fact that the histological grade was not reported in about $25 \%$ of the patients.

The search for the best treatment for retroperitoneal sarcomas still keeps us finding the balance based on the best evidence. In our career, we take into consideration the value and reference of the STRASS study, while believing in the benefits of irradiative treatments in retroperitoneal sarcomas. At least in the weekly multidisciplinary sarcoma tumor boards at our institution, we maintain this strategy to treat the patients who come to us. This debate is not over yet.

\section{AUTHORS" CONTRIBUTIONS}

SAH: Conceptualization, Data curation, Formal Analysis, Funding acquisition, Investigation, Methodology, Project administration, Resources, Software, Supervision, Validation, Visualization, Writing - original draft, Writing - review \& editing. ARNSS: Data curation, Formal Analysis, Funding acquisition, Investigation, Methodology, Project administration, Resources, Software, Visualization, Writing - original draft. TYTS: Data curation, Formal Analysis, Funding acquisition, Investigation, Methodology, Project administration, Resources, Software, Visualization, Writing - original draft. FCFR: Data curation, Formal Analysis, Funding acquisition, Investigation, Methodology, Project administration, Resources, Software, Visualization, Writing - original draft. DRFN: Data curation, Formal Analysis, Funding acquisition, Investigation, Methodology, Project administration, Resources, Software, Visualization, Writing - original 
draft. LHB: Data curation, Formal Analysis, Funding acquisition, Investigation, Methodology, Project administration, Resources, Software, Visualization, Writing - original draft. APAP: Data curation, Formal Analysis, Funding acquisition, Investigation, Methodology, Project administration, Resources, Software, Visualization, Writing - original draft. JLFS: Data curation, Formal Analysis, Funding acquisition, Investigation, Methodology, Project administration, Resources, Software, Visualization, Writing - original draft, Writing - review \& editing. MTMS: Data curation, Formal Analysis, Funding acquisition, Investigation, Methodology, Project administration, Resources, Software, Supervision, Validation, Visualization, Writing - original draft, Writing - review \& editing.

\section{REFERENCES}

1. Bonvalot S, Gronchi A, Le Péchoux C, Swallow CJ, Strauss $D$, Meeus $P$, et al. Preoperative radiotherapy plus surgery versus surgery alone for patients with primary retroperitoneal sarcoma (EORTC-62092: STRASS): a multicentre, open-label, randomised, phase 3 trial. Lancet Oncol. 2020;21(10):136677. https://doi.org/10.1016/S1470-2045(20)30446-0

2. Sindelar WF, Kinsella TJ, Chen PW, DeLaney TF, Tepper $J E$, Rosenberg SA, et al. Intraoperative radiotherapy in retroperitoneal sarcomas. Final results of a prospective, randomized, clinical trial. Arch Surg. 1993;128(4):402-10. https://doi.org/10.1001/archsurg.1993.01420160040005

3. Nussbaum DP, Rushing CN, Lane WO, Cardona DM, Kirsch DG, Peterson BL, et al. Preoperative or postoperative radiotherapy versus surgery alone for retroperitoneal sarcoma: a case-control, propensity score-matched analysis of a nationwide clinical oncology database. Lancet Oncol. 2016;17(7):966-75. https://doi.org/10.1016/S14702045(16)30050-X

4. Canter RJ, Martinez SR, Tamurian RM, Wilton M, Li CS, Ryu $J$, et al. Radiographic and histologic response to neoadjuvant radiotherapy in patients with soft tissue sarcoma. Ann Surg
Oncol. 2010;17(10):2578-84. https://doi.org/10.1245/s10434010-1156-3

5. Shah D, Borys D, Martinez SR, Li CS, Tamurian RM, Bold RJ, et al. Complete pathologic response to neoadjuvant radiotherapy is predictive of oncological outcome in patients with soft tissue sarcoma. Anticancer Res. 2012;329(9):3911-5. PMID: 22993336

6. Surgery with our without neoadjuvant chemotherapy in high risk retroperitoneal sarcoma (STRASS2). ClinicalTrials. gov NCT04031677. Available from: http://clinicaltrials.gov/ ct/show/NCT04031677.

7. O'Sullivan B, Davis AM, Turcotte R, Bell R, Catton C, Chabot $P$, et al. Preoperative versus postoperative radiotherapy in soft-tissue sarcoma of the limbs: a randomised trial. Lancet. 2002;359(9325):2235-41. https://doi.org/10.1016/S01406736(02)09292-9.

8. Shishak S, Rastogi S, Kalra K, Parisa M. STRASS trial: preoperative radiotherapy in retroperitoneal sarcoma: a commentary. Can Res Ther [Epub ahead of print] [cited 2020 Nov 16] Available from: https://www.cancerjournal.net/preprintarticle. asp?id=298077. https://doi.org/10.4103/jcrt.JCRT_573_19 